\title{
Preparation of polyhedral oligomeric silsesquioxane based cross-linked inorganic-organic nanohybrid as adsorbent for selective removal of acidic dyes from aqueous solution
}

Jiawei Liu*, Heng Yu, Qingmei Liang, Yanna Liu, Jiwei Shen, Quan Bai

Key Laboratory of Synthetic and Natural Functional Molecule Chemistry of the Ministry of Education, College of Chemistry \& Materials Science, Northwest University, Xi'an, 710127, P. R. China

\begin{abstract}
A cross-linked polyhedral oligomeric silsesquioxane (POSS) nanohybrid is synthesized through Schiff base chemistry by using octa-amino POSS as building blocks and glutaraldehyde as dual functional reagent. The obtained nanohybrid is characterized by FT-IR, solid-state ${ }^{29} \mathrm{Si}$ NMR, SEM, TEM, TGA and $\mathrm{N}_{2}$ adsorption-desorption isotherm, and the results demonstrate the successful cross-linkage between POSS molecules, forming block-like irregular nanoparticles with a specific surface area of $42.8 \mathrm{~m}^{2} \mathrm{~g}^{-1}$. As a novel solid-phase adsorbent, cross-linked POSS nanohybrid exhibits selective adsorption properties for acidic dyes, i.e., methyl orange (MO) in the present case. The strong electrostatic interactions between MO species and the nanohybrid are the main driving forces for MO adsorption. The kinetic data are well described by the pseudo-second-order kinetic model. The adsorption isotherm studies demonstrate that the data are well fit with
\end{abstract}


Langmuir model at $293 \mathrm{~K}$ and a maximum adsorption capacity of $237.5 \mathrm{mg} \mathrm{g}^{-1}$ is obtained. Thermodynamic studies indicate that the adsorption of MO is spontaneous and exothermal, along with reduced randomness. The adsorbed MO species could be effectively recovered by using methanol/ $\mathrm{NaOH}\left(9 / 1\right.$, v/v, $\left.0.1 \mathrm{~mol} \mathrm{~L}^{-1} \mathrm{NaOH}\right)$ as eluent. The practical application for textile wastewater treatment demonstrates that cross-linked POSS nanohybrid appears to be a promising material in dye removal field. Key words: polyhedral oligomeric silsesquioxane; cross-linked; acidic dye; adsorption and removal 


\section{Introduction}

With the development of dyestuff industry, a number of synthetic dyes have been widely used in textile, leather, paper, plastic, cosmetics, pharmaceutical, printing, and food processing [1]. However, environmental pollution caused by dyeing industrial effluents has been a worldwide problem as most of synthetic dyes are toxic and non-biodegradable [2]. The released dyes in water could lead to a serious hazard to aquatic life through affecting photosynthetic activity of aquatic plants [3]. Moreover, dyes and their metabolites will increase a carcinogenic risk for human and other animals [4,5]. Therefore, the sufficient removal of dyes prior to discharge of wastewater is of great important and many efforts have been made to achieve this aim. Up to now, various methods and technologies, such as chemical oxidation, photochemical degradation, coagulation/flocculation, biological treatment, membrane filtration and adsorption processes, have been developed to remove dyes from water [6-11]. In particular, adsorption is considered as an effective and economic method due to its high efficiency, low cost, simple operation, and favorable reusability $[3,12]$.

For adsorption process, the type and property of adsorbent might be the key factor that affects efficient dye adsorption and removal from aqueous solution. Therefore, the development of novel adsorbents dominates the applications of adsorption approach for dye removal. Recently, huge amount of attentions have been paid to inorganic-organic hybrid materials which combine advantages of both inorganic and 
organic components through incorporation of inorganic molecules into organic materials [13]. Polyhedral oligomeric silsesquioxane (POSS), usually considering as a cornerstone or scaffold for construction of inorganic-organic hybrid materials, consists of a defined chemical composition of $\mathrm{R}_{8} \mathrm{Si}_{8} \mathrm{O}_{12}$. The $\mathrm{Si}-\mathrm{O}-\mathrm{Si}$ skeleton is arranged into a cage-like inorganic core with a $\mathrm{T}_{8}$ cubic structure, and the $\mathrm{R}$ groups located at the eight vertexes of the cage are usually organic moieties, e.g., alkylene, aromatic, amino, and among others $[14,15]$. POSS owns a characteristic nanoscopic size of from 1 to $3 \mathrm{~nm}$ in diameter, which is comparable to the dimensions of polymeric segments in the condensed phase [15]. Therefore, POSS nanohybrid could be employed as building block for incorporation into polymers to effectively improve the physical, thermal, and/or mechanical properties of polymers, and the prepared POSS-based polymeric composites were used as photoelectrical materials, flame retardant, medical biomaterials, and chromatographic matrix [16-19] for various applications. In addition, the reactive/non-reactive organic groups as well as the hierarchical structure of POSS nanohybrid [20] further provide tremendous potentials for preparation of highly ordered materials [13,21] and multivalent bioconjugates [22-24], which could be applied for lectin binding [22], MRI monitoring [23], drug delivery [23,24], and so on. The high biodegradability and low toxicity make POSS-based bioconjugates as ideal bio-probes in biological and biomedical fields [23].

As one of representative POSS nanohybrids, octa-amino POSS (OA-POSS) exists eight primary amine groups in a form of chloride salts, which endows feasibility for 
surface modification and polymerization. For instance, OA-POSS were employed as ligand for preparation of CdSeTe quantum dots [25] and carbon dots [26]. Besides, water-soluble inorganic-organic hybrid gels/networks could be easily fabricated by covalent modification of OA-POSS with specific ligands that showed high selectivity towards glutathione (GSH) [27] and nucleoside triphosphates [28], respectively. In general, the eight amino moieties in OA-POSS not only possess reactivity for surface modification, but also offer abundant binding sites for adsorption. However, its super high water solubility makes the phase separation very difficult, thus limiting the application of OA-POSS as novel adsorbent for dye adsorption and removal. Cross-linking reaction between POSS particles through introducing a hydrophobic organic/polymeric reagent as bridge might be an effective strategy to overcome this problem, and this approach has been successfully used for synthesis of porous hybrid materials [29-31]. The POSS cage-like characteristic could be maintained after cross-linking reaction and the residual reactive groups showed possibility for further modification [30]. Nevertheless, to the best of our knowledge, the synthesis of cross-linked OA-POSS hybrid and its application as a novel adsorbent for dye adsorption and removal have never been reported.

In this work, we develop a simple and facile synthesis approach in aqueous solution for preparation of cross-linked POSS nanohyrid (Scheme 1). OA-POSS plays as the nanobuilding blocks and glutaraldehyde is employed as dual functional bridge for linkage of POSS molecules through well-known Schiff base chemistry [31]. 
Furthermore, the prepared cross-linked POSS nanohybrid is employed as a novel adsorbent for adsorption of acidic dyes, i.e., methyl orange (MO) in this particular case. Investigations on the adsorption behaviors of cross-linked POSS nanohybrid toward MO, including adsorption kinetics, isotherms as well as thermodynamics, are carried out in detail. In addition, the desorption and reusability of the nanohybrid are further evaluated. Finally, cross-linked POSS nanohybrid is applied for effective removal of dyes from real dyeing wastewater.

\section{Experimental}

\subsection{Materials and reagents}

3-aminopropyltriethoxysilane (APTS) and rhodamine $6 \mathrm{G}$ are purchased from Aladdin Reagent Co. Ltd, China. Methyl orange, congo red, methylene blue, glutaraldehyde ( $25 \%$ solution), methanol, acetonitrile and sodium borohydride $\left(\mathrm{NaBH}_{4}\right)$ are obtained from Sinopharm Chemical Reagent Co. Ltd (Shanghai, China). Hydrochloric acid (35 38\%) and sodium hydroxide are supplied by Bodi Chemical Holding Co. Ltd, (Tianjin, China). All the reagents are used as received without further purification. Ultra-high pure water with resistivity of $18 \mathrm{M} \Omega \mathrm{cm}$ is used throughout.

\subsection{Synthesis of OA-POSS}

The OA-POSS is prepared based on the previous work [26]. Typically, $20 \mathrm{~mL}$ of APTS and $160 \mathrm{~mL}$ of methanol are mixed together in a dried $250-\mathrm{mL}$ round-bottom flask and stirred homogenously. Then $27 \mathrm{~mL}$ of hydrochloric acid is added and the 
mixture is further stirred at room temperature for several days. Afterwards, the white precipitate is collected by centrifugation at $11000 \mathrm{rpm}$ for $10 \mathrm{~min}$, and then carefully washed by use of methanol for three times to remove any residual $\mathrm{HCl}$ and by-product. Finally, the white powder is obtained after drying at $60{ }^{\circ} \mathrm{C}$ for $24 \mathrm{~h}$ and grinding in a agate mortar.

\subsection{Preparation of cross-linked POSS nanohybrid}

The OA-POSS molecules are cross-linked by glutaraldehyde via Schiff base chemistry. Briefly, $1.0 \mathrm{~g}$ of as prepared OA-POSS powder is dissolved in $106.5 \mathrm{~mL}$ of water under continuous stirring to obtain a clear solution, and the $\mathrm{pH}$ value of solution is adjusted to 8.5 by $1.0 \mathrm{~mol} \mathrm{~L}^{-1} \mathrm{NaOH}$. After that, $2.175 \mathrm{~mL}$ of glutaraldehyde $(5.79$ mmol) is added dropwise into the mixture and the cross-linking reaction is carried out at room temperature for $2 \mathrm{~h}$ in dark place. Furthermore, $1.0915 \mathrm{~g}$ of $\mathrm{NaBH}_{4}(28.85$ mmol, dissolved in $5 \mathrm{~mL} \mathrm{H}_{2} \mathrm{O}$ ) is carefully added under vigorous stirring in an ice bath and the mixture is kept stirring for further $1 \mathrm{~h}$ at room temperature. After the reaction being accomplished, the product is collected by centrifugation at $11000 \mathrm{rpm}$ for $5 \mathrm{~min}$, followed by washing with water for three times and drying at $60{ }^{\circ} \mathrm{C}$ for $24 \mathrm{~h}$. A light-yellow powder is obtained after grinding in a agate mortar.

\subsection{Characterization}

Fourier transform infrared (FT-IR) spectra of OA-POSS and cross-linked POSS nanohybrid are measured by a TENSOR 27 FT-IR spectrometer (Bruker, Germany) 
from 400 to $4000 \mathrm{~cm}^{-1}$. The samples are prepared by following $\mathrm{KBr}$ pellet method. Solid-state ${ }^{29} \mathrm{Si}$ NMR spectra of POSS before and after cross-linking process are performed on a AVANCE III $400 \mathrm{WB}$ spectrometer (Bruker, Germany). The dried and finely powdered samples are packed into the $\mathrm{ZrO}_{2}$ rotor closed with Kel-F cap which is spun at $8 \mathrm{kHz}$ rate. The morphology of cross-linked POSS nanohybrid is recorded on an EVO18 scanning electronic microscopy (SEM, ZEISS, Germany). TEM images are obtained through HT7700 transmission electron microscope (Hitachi, Japan). The thermogravimetric analysis (TGA) is carried out by a TGA/DSC 1 STAR ${ }^{\mathrm{e}}$ System (Mettler-Toledo, Switzerland) from 25 to $800{ }^{\circ} \mathrm{C}$ with a heating rate of $10{ }^{\circ} \mathrm{C} \mathrm{min}{ }^{-1}$. The nitrogen adsorption-desorption isotherm of cross-linked POSS nanohybrid is obtained at $-196{ }^{\circ} \mathrm{C}$ by using a TriStar II 3020 specific surface area and porosity analyzer (Micromeritics Instrument Corporation, USA). The sample is degassed at 140 ${ }^{\circ} \mathrm{C}$ under vacuum for $11 \mathrm{~h}$ prior to the measurement.

\subsection{Adsorptive procedure}

Herein, methyl orange (MO) is employed as the model acidic dye for investigation of its adsorption and desorption behaviors on cross-linked POSS nanohybrid. Generally, cross-linked POSS nanohybrid $(5.0 \mathrm{mg})$ is added into a $\mathrm{MO}$ aqueous solution (100 $\mathrm{mg} \mathrm{L}^{-1}, 3 \mathrm{~mL}$ ) and the mixture is shaken vigorously for $60 \mathrm{~min}$ to facilitate the adsorption process. Then the supernatant is collected by centrifugation at $10000 \mathrm{rpm}$ for $5 \mathrm{~min}$, and the residual MO left in the original solution is further quantified by use of a UV-2550 UV-Vis spectrophotometer (Shimadzu, Japan) at 420 
$\mathrm{nm}$. The adsorption efficiency (E) and adsorption capacity (q) of MO on cross-linked POSS nanohybrid are calculated by Eq. (1) and (2), respectively, as follows:

$$
\begin{gathered}
E=\frac{\left(C_{0}-C_{e}\right)}{C_{0}} \times 100 \% \\
q=\frac{\left(C_{0}-C_{e}\right) \times V}{W}
\end{gathered}
$$

whereas $C_{0}$ and $C_{e}$ represent the original and residual MO concentration, respectively, $V$ represents the volume of MO solution, and $\mathrm{W}$ represents the weight of cross-linked POSS nanohybrid used during adsorption process.

The retained MO molecules are further recovered from the surface of cross-linked POSS nanohybrid by using a mixture of methanol and $\mathrm{NaOH}$ solution $(9 / 1, \mathrm{v} / \mathrm{v}, 0.1$ mol $\left.\mathrm{L}^{-1} \mathrm{NaOH}\right)$ as eluent. The desorption process is performed under vigorous oscillation for $90 \mathrm{~min}$. Then after centrifugation at $10000 \mathrm{rpm}$ for $5 \mathrm{~min}$, the supernatant is collected for the ensuing investigations.

\section{Results and Discussion}

\subsection{Characterization of cross-linked POSS nanohybrid}

Herein, we develop a simple and facile approach for preparation of cross-linked POSS nanohybrid in aqueous solution at room temperature. As shown in Scheme 1, the hydrochloride salts of OA-POSS are firstly neutralized by adjusting $\mathrm{pH}$ value of aqueous solution from 3 to 8.5, so that the bare amino groups could be exposed for the following cross-linking reaction. After that, glutaraldehyde is adopted as the 
cross-linking reagent for conjugation of POSS molecules based on Schiff base chemistry. Finally, the excess glutaraldehyde is reduced by $\mathrm{NaBH}_{4}$, and cross-linked POSS nanohybrid is obtained at the same time. In this work, OA-POSS is employed as the nanobuilding block for construction of cross-linked nanohybrid. Its intrinsic cage-like skeleton plays as the basic matrix of this nanohybrid, and the reactive amino groups provide abundant binding sites for both cross-linking reaction and dye adsorption. On the other hand, glutaraldehyde is used as a mild dual functional bridge for densely connection of POSS molecules in aqueous solution. Moreover, the alkyl chains of glutaraldehyde could increase the insolubility of cross-linked POSS nanohybrid, which facilitates the operation of solid-liquid separation.

The conversions of functional groups of OA-POSS during the cross-linking process are monitored by FT-IR spectra, as illustrated in Fig. 1A. For OA-POSS, three characteristic absorption bands at 3020,1580 and $1505 \mathrm{~cm}^{-1}$ are assigned to the $-\mathrm{NH}_{3}{ }^{+}$ groups, indicating that a hydrochloride salt of OA-POSS is formed during the hydrolysis process. The obvious stretching vibration peak of Si-O-Si groups at around $1120 \mathrm{~cm}^{-1}$ is derived from the cage-like silica skeleton [31]. In the spectrum of cross-linked POSS nanohybrid, the typical adsorption bands of $-\mathrm{NH}_{3}{ }^{+}$groups disappear, while the bands attributed to secondary amine groups (NH) at 3400 (stretching, strong) and $1560 \mathrm{~cm}^{-1}$ (bending, weak) are found. This conversion demonstrates that the hydrochloride salts are effectively neutralized and the exposed $\mathrm{NH}_{2}$ groups are conjugated with glutaraldehyde. Moreover, the absent stretching vibration band at 
$\sim 1725 \mathrm{~cm}^{-1}$ of carbonyl moieties $(\mathrm{C}=\mathrm{O})$ from aldehydes, as well as the presence of three bands at 2935,2865 and $1460 \mathrm{~cm}^{-1}$ corresponding to $-\mathrm{CH}_{2}$ moieties, further indicate that glutaraldehydes are completely converted through Schiff base chemistry. The observation of C-N bonds (stretching vibration at $1340 \mathrm{~cm}^{-1}$ ) suggests that the formed $\mathrm{C}=\mathrm{N}$ imine groups between aldehydes and amines have been sufficiently reduced by $\mathrm{NaBH}_{4}$. Nevertheless, the bending vibration at $1650 \mathrm{~cm}^{-1}$ attributed to primary amine reveals the presence of residual $\mathrm{NH}_{2}$ moieties in cross-linked POSS nanohybrid. Similar absorption bands of Si-O-Si skeleton at $1120 \mathrm{~cm}^{-1}$ for both OA-POSS and cross-linked POSS nanohybrid indicate that the cage-like structure of POSS is maintained during the cross-linking process.

Solid-state ${ }^{29} \mathrm{Si}$ NMR measurements are performed to investigate the structures of both OA-POSS and cross-linked POSS nanohybrid and the results are shown in Fig. 1B. A single strong resonance signal with the chemical shift at $-68.1 \mathrm{ppm}$ is observed for OA-POSS, revealing that there is only one structural conformation for all silicon atoms [32]. Besides, the similar value of chemical shift with that of the silicon atoms in the cage structure of $\left(\mathrm{RSiO}_{1.5}\right)_{8}$ confirms that the prepared OA-POSS has a symmetric cage structure [32]. The ${ }^{29} \mathrm{Si}$ NMR spectrum of cross-linked POSS nanohybrid shows a main resonance peak at $-68.1 \mathrm{ppm}$ with a shoulder at $-60.9 \mathrm{ppm}$, which can be categorized into two kinds of silicon atoms with different substitution circumstances, i.e., the linkage with formed secondary amine groups and residual primary amine groups, respectively. As both of the signals fall in the typical range of POSS cage 
structure [31], cross-linked POSS nanohybrid owns the same structural feature with that of OA-POSS. Solid-state ${ }^{29} \mathrm{Si}$ NMR results demonstrate that the cage structure of POSS is retained during the cross-linking process.

The surface morphology of cross-linked POSS nanohybrid is obtained by SEM image (Fig. 2A). It is seen that the prepared nanohybrid consists of plenty of block-like particles with irregular shapes and rough surfaces. The average particle size is estimated to be around $120 \mathrm{~nm}$ with a distribution of 70-200 nm. The inner structure of cross-linked POSS nanohybrid is determined by TEM image. As shown in Fig. 2B, the nanohybrid displays full filled particles without any porous or hollow structures. This demonstrates that OA-POSS molecules are densely connected together by glutaraldehyde. An average particle size of $c a .110 \mathrm{~nm}$ is obtained from TEM image, which is well in accordance with that from SEM results. Moreover, the poor dispersion and serious stacking of cross-linked POSS nanohybrid in both SEM and TEM images indicate that the insolubility of the nanohybrid significantly increases after cross-linking reaction.

Fig. S1A illustrates the $\mathrm{N}_{2}$ adsorption-desorption isotherm of cross-linked POSS nanohybrid. Based on the IUPAC classification, the isotherm of the nanohybrid appears to show normal Type II adsorption that is usually observed with non-porous or macroporous adsorbents [33]. A specific surface area of $42.8 \mathrm{~m}^{2} \mathrm{~g}^{-1}$ for cross-linked POSS nanohybrid is further obtained under BET (Brunauer-Emmett-Teller) method by use of the adsorption data in the range of $\mathrm{P} / \mathrm{P}_{0}$ from 0.05 to 0.35 . Comparing to the 
previous work [30,31], the low surface area and nonporous property of cross-linked POSS nanohybrid might be ascribed to the utilization of short-chain aldehyde reagents rather than long-chain polymers for cross-linking reaction. Nevertheless, in the present case, the surface area is large enough for adsorption of acidic dyes from aqueous solution. Furthermore, the thermal properties of OA-POSS and cross-linked POSS nanohybrid are investigated and the results are seen in Fig. S1B. OA-POSS exhibits high thermal stability under $350{ }^{\circ} \mathrm{C}$, and the main weight losses occurred in the range of $350-425{ }^{\circ} \mathrm{C}$ and $425-700{ }^{\circ} \mathrm{C}$ are derived from the degradation of aminopropyl groups and Si-O-Si skeleton, respectively (Fig. S1B(a)). For cross-linked POSS nanohybrid, in addition to the similar thermolysis of POSS frameworks between $350-700{ }^{\circ} \mathrm{C}$ as OA-POSS, a new weight loss stage within $100-350{ }^{\circ} \mathrm{C}$ is appeared due to the decomposition of carbon chain skeleton derived from glutaraldehyde molecules (Fig. S1B(b)). Thermal analysis results demonstrate that cross-linked POSS nanohybrid is successfully prepared under the present cross-linking approach.

\subsection{Effect of various parameters on MO adsorption process}

In this work, the effect of cross-linked POSS nanohybrid dosage on both the adsorption efficiency and adsorption capacity of MO is firstly investigated by varying the adsorbent dosage ranged from 3.0 to $30.0 \mathrm{mg}$ with a constant initial concentration of MO at $100 \mathrm{mg} \mathrm{L}^{-1}$. The results are illustrated in Fig. S2A. An obvious increase of adsorption efficiency of MO from $c a$. $20 \%$ to $90 \%$ is obtained by increasing adsorbent dosage from 3 to $10 \mathrm{mg}$. This is attributed to the increase of surface area as well as 
more available adsorption sites of the nanohybrid [3]. With further increasing the sorbent dosage, there is no significant change of adsorption efficiency and a maximum value of $c a .95 \%$ is achieved. In contrast, the adsorption capacity of MO onto the nanohybrid $(q)$ rapidly decreases from 90 to $10 \mathrm{mg} \mathrm{g}^{-1}$ when the adsorption dosage increases from 3 to $30 \mathrm{mg}$. The decrease of adsorption capacity is derived from the splitting effect of flux or concentration gradient between MO concentration in solution and MO adsorbed on the nanohybrid, thus resulting in the decline of $q$ value by increasing the adsorbent dosage [3]. Herein, considering both adsorption efficiency and capacity, the following experiments are carried out by use of $5 \mathrm{mg}$ sorbent, giving rise to a $q$ value of $c a .50 \mathrm{mg} \mathrm{g}^{-1}$ with an approximate efficiency of $85 \%$.

It is known that the structures and properties of dyes are highly sensitive toward the $\mathrm{pH}$ values of their circumstance, which might further affect the adsorption performances of dyes onto adsorbent surface. Besides, the surface property of sorbent also could be effectively changed by varying the $\mathrm{pH}$ values of solutions. Therefore, the effect of $\mathrm{pH}$ on $\mathrm{MO}$ adsorption is investigated and the results are shown in Fig. S2B. Generally, the adsorption capacity of MO on the nanohybrid gradually decreases with the increase of $\mathrm{pH}$ values. When $\mathrm{pH}$ value is 2 and 3 , the adsorption capacity of MO could reach $c a .60 \mathrm{mg} \mathrm{g}^{-1}$, which is higher than that of $\mathrm{MO}$ under other $\mathrm{pH}$ conditions. At $\mathrm{pH}$ range of 4-11, the adsorption capacity of $\mathrm{MO}$ almost keeps constant with a slight decline by increasing the $\mathrm{pH}$ values. While when the $\mathrm{pH}$ further increases to 12 , a sharply decrease of adsorption capacity is obtained, indicating that the surface of the 
nanohybrid is not suitable for MO adsorption under strong basic condition.

In order to understand the adsorption mechanism of MO molecules onto the nanohybrid, the surface charge properties of cross-linked POSS nanohybrid under various $\mathrm{pH}$ conditions are further investigated. As seen in Fig. S2C, the surface of the nanohybrid is highly positive charged (zeta potential is over $+20 \mathrm{mV}$ ) in a wide $\mathrm{pH}$ range of $2-10$, revealing that there are abundant residual amino groups on the surface of the nanohybrid. However, under strong basic condition $(\mathrm{pH}=11$ and 12$)$, zeta potential of the nanohybrid significantly decreases and even the surface converts into negatively charged. This might be ascribed to the partly destruction of POSS cage structure and the formation of silanol groups under basic condition [34]. It is interesting that the adsorption profile of $\mathrm{MO}$ on the sorbent is highly matched with the variation of surface charges of the nanohybrid under various $\mathrm{pHs}$, indicating that the MO adsorption is dominated by surface charge of the nanohybrid. In this case, methyl orange is a kind of azo-dye with a sulfonic group, which provides strong negative charges in the whole tested $\mathrm{pH}$ range (2-12). Consequently, the opposite surface charge properties of MO molecules and the nanohybrid offer strong electrostatic interactions for facilitating the adsorption process. When the $\mathrm{pH}$ is lower, the electrostatic attractions are strengthened so that the adsorption capacity is higher. While with the increase of $\mathrm{pH}$, the decrease of positive charges of the nanohybrid weakens the electrostatic interactions and results in lower adsorption capacity. When the $\mathrm{pH}$ is high enough ( $\mathrm{pH}$ 12), both MO molecules and the nanohybrid are negatively charged, thus 
the electrostatic repulsions give rise to the significant decline of adsorption capacity. Although the largest adsorption capacity of $c a .60 \mathrm{mg} \mathrm{g}^{-1}$ is obtained at $\mathrm{pH} 3$, the solubility of MO in acidic solution is poor in particular with the increase of MO concentration (data are not shown), thus all the following adsorption experiments are carried out in neutral solution (pH 6).

Fig. S2D shows the effect of ionic strength on the adsorption of MO onto the nanohybrid by addition of different concentrations of $\mathrm{NaCl}$ into $\mathrm{MO}$ sample solutions. As seen that the adsorption capacity of MO significantly drops from 48 to $28 \mathrm{mg} \mathrm{g}^{-1}$ with the $\mathrm{NaCl}$ concentration increases from 0 to $0.2 \mathrm{~mol} \mathrm{~L}^{-1}$. The addition of $\mathrm{NaCl}$ trends to destroy the electric double layers formed at the interface of the nanohybrid and bulk solution, meanwhile the added salt partly neutralizes the charges of the sorbent binding sites [35], thus weakening the electrostatic interactions between MO and the nanohybrid, and deteriorating the adsorption performance. While with higher $\mathrm{NaCl}$ concentration $\left(>0.5 \mathrm{~mol} \mathrm{~L}^{-1}\right)$, the adsorption capacity is not further declined, in contrast, it exhibits tendency to be increased. This indicates that there might be other driving forces contributed to MO adsorption. It should be considered that the Si-O-Si skeleton of POSS is strong hydrophobic and its hydrophobicity could be strengthened through junction units [20]. Meanwhile, MO contains two aromatic rings, which could provides hydrophobic interactions with the nanohybrid. High ionic strength would enhance the hydrophobic interactions [36] and facilitate MO adsorption. Overall, in the present case, no salt is added during the adsorption process. 
According to the effect results of both $\mathrm{pH}$ and ionic strength, electrostatic interactions are considered as the main driving force for $\mathrm{MO}$ adsorption while hydrophobic interactions are subordinate. For further verifying this adsorption mechanism, another three kinds of dyes, i.e., congo red (CR), rhodamine 6G (R6G) and methylene blue (MB) are employed for investigating their adsorption behaviors on cross-linked POSS nanohybrid. The adsorption experiments are carried out under optimized conditions and the results are shown in Fig. S3A. It is clear that CR and MO could be effectively adsorbed onto the nanohybrid, while R6G and MB show very poor adsorption performances on this adsorbent. This indicates that cross-linked POSS nanohybrid shows high selectivity towards acidic dyes. As seen in Fig. S3B, both CR and MO molecules contain negatively charged sulfonic groups, which could provide strong electrostatic interactions with the positively charged nanohybrid to achieve high adsorption capacities. However, there is no negatively charged groups found in R6G and $\mathrm{MB}$, in contrast, the positively charged moieties $\left(=\mathrm{N}^{+}\right)$might hinder their adsorption onto the nanohybrid through electrostatic repulsion forces. This result demonstrates that electrostatic interactions make acidic dyes be selectively adsorbed onto cross-linked POSS nanohybrid (Scheme 1).

\subsection{Adsorption kinetic studies}

For the purpose of achieving equilibrium adsorption and maximum capacity, the effect of contact time on MO adsorption capacity is investigated at three different concentrations of 50, 100 and $200 \mathrm{mg} \mathrm{L}^{-1}$. As seen in Fig. 3, all the adsorption kinetic 
curves display rapid increase in the initial period ( $<15 \mathrm{~min})$, which might be attributed to the availability of a large number of vacant sites on the adsorbent surface [12]. With the further increase of adsorption time (15-60 $\mathrm{min})$, the increase of adsorption capacity is clearly decelerated because of the decrease of vacant binding sites. An equilibrium plateau is eventually reached at $c a .60 \mathrm{~min}$ and no marked increase is observed when contact time reaches $120 \mathrm{~min}$. The decrease of adsorption rate is due to the reduction in the concentration gradient of MO between the aqueous and solid phases.

In order to investigate the adsorption mechanism and determine the kinetic parameters of the MO adsorbed onto cross-linked POSS nanohybrid, three kinetic models, i.e., pseudo-first-order, pseudo-second-order, and intra-particle diffusion kinetic models, are applied to fit the experiment data. The pseudo-first-order model is described by Lagergren [3] and its linear form can be expressed as Eq. (3):

$$
\ln \left(q_{e}-q_{t}\right)=\ln q_{e}-\frac{k_{1} t}{2.303}
$$

where $q_{e}$ and $q_{t}$ are the adsorption capacity $\left(\mathrm{mg} \mathrm{g}^{-1}\right)$ of MO at equilibrium and at time $\mathrm{t}$ (min), respectively; $k_{l}$ is the pseudo-first-order rate constant $\left(\mathrm{min}^{-1}\right)$; and $t$ is the contact time (min). The $k_{l}$ value could be calculated from the slope of the plot of $\ln \left(q_{e}-q_{t}\right)$ versus $t$.

The adsorption kinetics also could be described by pseudo-second-order model [12] as the following linear equation (4):

$$
\frac{t}{q_{t}}=\frac{1}{k_{2} \times q_{e}^{2}}+\frac{t}{q_{e}}
$$

where $k_{2}$ is the pseudo-second-order rate constant $\left(\mathrm{g} \mathrm{mg}^{-1} \mathrm{~min}^{-1}\right)$. The value of $k_{2}$ and $q_{e}$ 
can be determined by the plot of $t / q_{t}$ versus $t$. The calculated kinetic parameters from two models are listed in Table 1 and the corresponding curves are illustrated in Fig. S4. As seen in Table 1 , the correlation coefficient values $\left(R^{2}\right)$ obtained according to the pseudo-first-order kinetic model are rather low, indicating that the adsorption process could not be described by this model. However, it is found that all the experiment data show favorable compliance with pseudo-second-order kinetic model in terms of high correlation coefficient values $\left(R^{2}>0.999\right)$, which suggests that the pseudo-second-order model is more appropriate to describe the adsorption behavior of MO onto the nanohybrid. Moreover, minor derivations between the calculated and the experimental $q_{e}$ values from the pseudo-second-order model also demonstrate that MO adsorption onto the nanohybrid ideally follows the pseudo-second-order kinetic model.

For a solid-liquid adsorption process, the mass transfer of adsorbate is usually dominated by external mass transfer, or intra-particle diffusion, or both. To identify the mechanism involved in the adsorption process, the intra-particle diffusion model [2] is employed as follows:

$$
q_{t}=k_{i d} t^{0.5}+A
$$

where $k_{i d}\left(\mathrm{mg} \mathrm{g}^{-1} \min ^{-0.5}\right)$ is the intraparticle diffusion rate constant and $A\left(\mathrm{mg} \mathrm{g}^{-1}\right)$ represents the thickness of the boundary layer. As seen in Fig. S5, each plot of $q_{t}$ versus $t^{0.5}$ at different initial MO concentration exhibits a multilinearity relation and could be separated into two distinct linear regions. This indicates that the adsorption process is influenced by two or more steps. The first linear portion occurred from 0 to $4 \mathrm{~min}^{0.5}$ for 
all three concentrations is related to external surface adsorption or instantaneous adsorption [37]. This might be ascribed to boundary layer adsorption of adsorbent or diffusion of adsorbate from bulk solution to the surface of the adsorbent [3]. The second gentle-slope linear portion represents the gradual adsorption stage, which is controlled by intra-particle diffusion. This step is attributed to diffusion of the adsorbate from the exterior surface to the pores of the adsorbent internal structure [12].

The $k_{i d}$ and $A$ values at each MO concentration are listed in Table S1. It is clearly observed that the diffusion rate constants for all MO concentrations follow the order of $k_{i d 1}>k_{i d 2}$, suggesting that the first diffusion step is the fastest stage [1]. Furthermore, none of the linear segments passes through the origin $(A>0)$, demonstrating that intra-particle diffusion is not the unique rate-limiting step, and the adsorption rate might be controlled by some other processes [3].

\subsection{Adsorption isotherm studies}

Adsorption isotherm curve reveals the relationship between the concentration of solutes in solution and that on adsorbent under equilibrium state at certain temperature. In this case, adsorption isotherms of MO on the nanohybrid at various temperatures are carried out by performing the adsorption process at $\mathrm{pH} 6$ within a wide range of initial MO concentration (10-700 $\left.\mathrm{mg} \mathrm{L}^{-1}\right)$. The results are illustrated in Fig. 4. It is seen that the amount of MO adsorbed on the nanohybrid gradually increases with the increment of its initial concentration, and a saturation adsorption trends to reach when the concentration is over $600 \mathrm{mg} \mathrm{L}^{-1}$. However, improving the temperature deteriorates the 
adsorption of MO on the nanohybrid, resulting in lower adsorption capacity.

For further evaluating adsorption isotherms of MO species onto the nanohybrid, Langmuir, Freundlich and Dubinin-Radushkevich isotherm models are tested with the experimental data. Langmuir theoretical model assumes that monolayer adsorption occurs on a homogenous sorbent surface with numbers of identical adsorption sites [38]. The linear form of Langmuir isotherm equation is described as follows:

$$
\frac{C_{e}}{q_{e}}=\frac{1}{b \times q_{m}}+\frac{C_{e}}{q_{m}}
$$

where $C_{e}\left(\mathrm{mg} \mathrm{L}^{-1}\right)$ is the equilibrium concentration of $\mathrm{MO}$ in the bulk solution, $q_{e}(\mathrm{mg}$ $\left.\mathrm{g}^{-1}\right)$ is the equilibrium adsorption capacity of cross-linked POSS nanohybrid, $b\left(\mathrm{~L} \mathrm{mg}^{-1}\right)$ is the equilibrium constant, $q_{m}\left(\mathrm{mg} \mathrm{g}^{-1}\right)$ is the maximum adsorption capacity of the nanohybrid when adsorption achieves monolayer coverage.

Table 2 lists the values of $q_{m}$ and $b$ at 293, 303, 313, 323 and $333 \mathrm{~K}$, respectively. They are calculated from the slope and intercept data of the plots of $C_{e} / q_{e}$ versus $C_{e}$, and these plots are drawn based on the experimental isotherm data of MO adsorbed onto the nanohybrid (the plots are shown in Fig. S6A). As seen in Table 2, the experimental data are well in agreement with Langmuir model with a high correlation coefficient value $\left(R^{2}=0.9967\right)$ at $293 \mathrm{~K}$, and a maximum adsorption capacity of $c a$. $237.5 \mathrm{mg} \mathrm{g}^{-1}$ is obtained. However, lower adsorption capacities with worse linearity of the plots of $C_{e} / q_{e}$ versus $C_{e}$ are observed at higher temperature. When the temperature rises to $333 \mathrm{~K}$, the adsorption capacity significantly drops from 237.5 to $142.9 \mathrm{mg} \mathrm{g}^{-1}$ and the correlation coefficient value also declines to 0.9818. Moreover, the Langmuir 
constant, $b$, is found to be decreased from 0.0127 to $0.0111 \mathrm{~L} \mathrm{mg}^{-1}$ as well. These results indicate that the adsorption process of MO onto the nanohybrid ideally follows Langmuir model at room temperature, and with increase of temperature, less MO molecules are fixed onto the nanohybrid surface, leading to decrease of adsorption capacity.

Freundlich isotherm model is an empirical equation that assumes the adsorption process occurs on a heterogeneous sorbent surface and the adsorbates preferentially occupies the active affinity binding sites $[39,40]$. Its linear form can be written as:

$$
\ln q_{e}=\ln K_{F}+\frac{1}{n} \ln C_{e}
$$

where $q_{e}\left(\mathrm{mg} \mathrm{g}^{-1}\right)$ is the amount of solute adsorbed per unit weight of adsorbent, $C_{e}$ $\left(\mathrm{mg} \mathrm{L}^{-1}\right)$ is the equilibrium concentration of solute in the bulk solution, $K_{F}\left(\mathrm{mg}^{1-1 / n} \mathrm{~L}^{1 / \mathrm{n}}\right.$ $\mathrm{g}^{-1}$ ) is Freundlich constant that relates to the adsorption capacity, and $n$ is the adsorptive index which represents the interactions between adsorbate molecules and adsorbent surface. The plots of $\ln q_{e}$ versus $\ln C_{e}$ at various temperatures are drawn in Fig. S6B and the $K_{F}$ and $1 / n$ values are listed in Table 2. It is worth noting that all the curves of $\ln q_{e}$ versus $\ln C_{e}$ exhibit high correlation coefficient values $\left(\mathrm{R}^{2}>0.996\right)$, indicating that Freundlich model is quite reasonable for description of MO adsorption process onto cross-linked POSS nanohybrid under a wide range of temperature. Besides, better linearity of the plot is obtained at higher temperature, which implies the enhancement of the heterogeneity of the adsorbent surface. According to the theory, the $n$ value could provide a indication of the favorability and capacity of the 
adsorbent/adsorbate system, and $0.1<1 / n<0.5$ represents favorable adsorption process [41]. In this case, the values of $1 / n$ at different temperatures between 0.4-0.5 suggest that the adsorption behavior of MO onto the nanohybrid is favorable.

Dubinin-Radushkevich model is another important isotherm model based on the Polanyi potential theory and usually used to distinguish between physical or chemical adsorption [42]. The equation of D-R model is described as:

$$
q_{e}=q_{D-R} \exp \left(-K \varepsilon^{2}\right)
$$

where $K\left[(\mathrm{~mol})^{2}(\mathrm{~kJ})^{-2}\right]$ is a constant that relates to the free adsorption energy, $q_{D-R}(\mathrm{mg}$ $\left.\mathrm{g}^{-1}\right)$ is the maximum adsorption capacity, and $\varepsilon\left(\mathrm{kJ} \mathrm{mol}^{-1}\right)$ is Polanyi potential which can be calculated by Equation 9:

$$
\varepsilon=R T \ln \left(1+1 / C_{e}\right)
$$

where $C_{e}$ is the retained concentration of solute in the bulk solution, $R$ is the universal gas constant $\left(8.314 \mathrm{~J} \mathrm{~mol}^{-1} \mathrm{~K}^{-1}\right)$, and $T$ is the contact temperature $(\mathrm{K})$.

The mean free adsorption energy $(E)$ is calculated by use of $K$ value according to the following equation:

$$
E=(2 K)^{-1 / 2}
$$

where the value of $E$ predicts the type of adsorption process. Generally, if the value of $E$ is lower than $8 \mathrm{~kJ} \mathrm{~mol}^{-1}$, the adsorption process is physical, if the value is between 8 and $16 \mathrm{~kJ} \mathrm{~mol}^{-1}$, the process is dominated by chemical ion exchange adsorption, and if the value is more than $20 \mathrm{~kJ} \mathrm{~mol}^{-1}$, chemical adsorption should be involved [43]. The results of both $E$ values and calculated $q_{D-R}$ values are shown in Table 2 . It is seemed 
that the $E$ value of MO adsorbed onto the nanohybrid almost keeps constant between 6.71 to $6.98 \mathrm{~kJ} \mathrm{~mol}^{-1}$ under various temperatures, and the adsorption energy is lower than $8 \mathrm{~kJ} \mathrm{~mol}^{-1}$, indicating that adsorption process is controlled by physical binding force, i.e., electrostatic and hydrophobic interactions in the present case.

Furthermore, it should be noted that the correlation coefficient values $\left(\mathrm{R}^{2}=0.951-0.966\right)$ evaluated from $\mathrm{D}-\mathrm{R}$ model (the linear plots are shown in Fig. S6C) are much lower than those from the previous two models, indicating that D-R model shows poor fit of experimental data in this case. Overall, by comparing the results of this study, the most suitable isotherm models for MO adsorption on cross-linked POSS nanohybrid are determined in the order of Freundlich $>$ Langmuir > Dubinin-Radushkevich.

Table 3 lists a comparison of MO removal performances of cross-linked POSS nanohybrid with several adsorbents reported in previous studies. It is found that the adsorption/removal of MO from aqueous solution by use of cross-linked POSS nanohybrid could be accomplished in a short time $(60 \mathrm{~min})$ with a favorable or comparable adsorption capacity $\left(237.5 \mathrm{mg} \mathrm{g}^{-1}\right)$. Moreover, under the definite condition (fixed MO concentration and adsorbent dosage), a high MO removal efficiency of $85 \%$ is also obtained by cross-linked POSS nanohybrid. This result confirms that cross-linked POSS nanohybrid used in this study is a relatively suitable adsorbent in the field of dye removal.

\subsection{Adsorption thermodynamics}


Thermodynamic study could provide important parameters that associate with in-depth information regarding the nature and feasibility of the adsorption process. Four important parameters, i.e., adsorption equilibrium constant $\left(\mathrm{K}_{0}\right)$, Gibbs free energy change $\left(\Delta \mathrm{G}^{\theta}, \mathrm{kJ} \mathrm{mol}^{-1}\right)$, enthalpy change $\left(\Delta \mathrm{H}^{\theta}, \mathrm{kJ}^{\mathrm{mol}}{ }^{-1}\right)$, and entropy change $\left(\Delta \mathrm{S}^{\theta}, \mathrm{J} \mathrm{mol}^{-1} \mathrm{~K}^{-1}\right)$ for the adsorption of MO on cross-linked POSS nanohybrid are calculated according to equations $11-13$ [53,54]:

$$
\begin{gathered}
K_{0}=\frac{q_{e}}{C_{e}} \\
\Delta G^{\theta}=-R T \ln K_{0} \\
\ln K_{0}=\frac{\Delta S^{\theta}}{R}-\frac{\Delta H^{\theta}}{R T}
\end{gathered}
$$

where $q_{e}\left(\mathrm{mg} \mathrm{g}^{-1}\right)$ is the amount of MO species adsorbed on the per unit weight of cross-linked POSS nanohybrid, $C_{e}\left(\mathrm{mg} \mathrm{L}^{-1}\right)$ is the equilibrium concentration of $\mathrm{MO}$ in bulk solution, $T(\mathrm{~K})$ is the temperature tested in the experiment, $R$ is the universal gas constant $\left(8.314 \mathrm{~J} \mathrm{~mol}^{-1} \mathrm{~K}^{-1}\right)$. The values of $\mathrm{K}_{0}$ at various temperatures could be obtained by plotting $\ln \left(q_{e} / C_{e}\right)$ against $q_{e}$ and extrapolating $q_{e}$ to zero (the plots are illustrated in Fig. S7A) [53]. The values of $\Delta \mathrm{H}^{\theta}$ and $\Delta \mathrm{S}^{\theta}$ are calculated from the slop and intercept of a van't Hoff plot of $\ln K_{0}$ versus $1 / \mathrm{T}$ as presented in Fig. S7B.

The negative values of $\Delta \mathrm{G}^{\theta}\left(-5.24,-4.83,-4.54,-3.96\right.$ and $-3.71 \mathrm{~kJ} \mathrm{~mol}^{-1}$ corresponding to $293,303,313,323$, and $333 \mathrm{~K}$ ) indicate that the adsorption process of MO on the nanohybrid is spontaneous and feasible. However, the absolute value of $\Delta \mathrm{G}^{\theta}$ decreases with the increase of temperature, revealing that the spontaneity reduces at higher temperature. The values of $\Delta \mathrm{H}^{\theta}$ and $\Delta \mathrm{S}^{\theta}$ are determined to be $-16.80 \mathrm{~kJ} \mathrm{~mol}^{-1}$ 
and $-39.45 \mathrm{~J} \mathrm{~mol}^{-1} \mathrm{~K}^{-1}$, respectively. The former $\Delta \mathrm{H}^{\theta}$ value confirms adsorption process is exothermal and MO adsorption on the nanohybrid is favored at lower temperature. This result fully agrees with the fact that adsorption capacity of MO decreases with temperature observed in previous section. The latter negative $\Delta \mathrm{S}^{\theta}$ value implies the decreased randomness of MO species after being adsorbed on the surface of the nanohybrid.

\subsection{MO desorption and regeneration of the nanohybrid}

The desorption property of MO species from the surface of cross-linked POSS nanohybrid after adequate adsorption is carefully investigated to achieve effective MO release from the nanohybrid and facile regeneration of the sorbent. In this case, three different eluents, i.e., acetonitrile, methanol and mixture of methanol with $\mathrm{NaOH}$ aqueous solution $\left(9 / 1, \mathrm{v} / \mathrm{v}, 0.1 \mathrm{~mol} \mathrm{~L} \mathrm{~L}^{-1} \mathrm{NaOH}\right)$, are employed for desorption studies. The desorption processes are repeated four times for each kind of eluent to reach sufficient desorption. The total desorption efficiency for each eluent is the sum of efficiency values for four times, as illustrated in Fig. 5A. It is found that the total desorption efficiency of each eluent is $34.8 \%$ for acetonitrile, $63.0 \%$ for methanol, and $89.5 \%$ for methanol/ $\mathrm{NaOH}\left(9 / 1, \mathrm{v} / \mathrm{v}, 0.1 \mathrm{~mol} \mathrm{~L}^{-1} \mathrm{NaOH}\right)$, respectively. This indicates that the desorption abilities of these three eluents are determined in the order of acetonitrile $<$ methanol $<$ methanol/ $\mathrm{NaOH}$. In particular, after adding small amount of $\mathrm{NaOH}$ solution into methanol, the first desorption efficiency sharply increases from $28.6 \%$ to $83.9 \%$. This is attributed to the electrostatic repulsions between MO species 
and cross-linked POSS nanohybrid under basic condition, and a synergistic effect of methanol and $\mathrm{NaOH}$ results in the significant increase of desorption efficiency.

The reusability of cross-linked POSS nanohybrid for MO removal is tested by repeating adsorption and desorption processes for three times with the same sorbent. Adsorption process is carried out by using $3.0 \mathrm{~mL}$ of MO solution at $100 \mathrm{mg} \mathrm{L}^{-1}$, while regeneration of the sorbent is performed four times with methanol/ $\mathrm{NaOH}(9 / 1, \mathrm{v} / \mathrm{v}, 1$ mol $\mathrm{L}^{-1} \mathrm{NaOH}$ ) for each adsorption-desorption cycle. Fig. 5B shows the adsorbed amounts of MO species on the fresh $\left(1^{\text {st }}\right.$ column) and reused sorbent $\left(2^{\text {nd }}\right.$ and $3^{\text {rd }}$ columns). It is seen that a gradual decrease of adsorption capacity is observed after three adsorption-desorption cycles because of the incomplete desorption of MO for each time. Nevertheless, the high enough adsorption capacity of cross-linked POSS nanohybrid towards MO species after three successive adsorption-desorption cycles still demonstrates its potential reusability in practical applications.

The structural stability of cross-linked POSS nanohybrid after performing one or three adsorption-desorption cycles are characterized by FT-IR spectra and SEM images, as seen in Fig. S8. It is clearly observed that all the characteristic peaks of cross-linked POSS nanohybrid after one or three adsorption-desorption cycles are located at the same positions as those in the FT-IR spectrum of fresh cross-linked POSS nanohybrid, indicating that all the functional groups are maintained during the adsorption-desorption processes (Fig. S8A). Moreover, according to the SEM imaging results, the similar surface morphologies and particle sizes of all the three nanohybrids 
further demonstrate that the basic structure of cross-linked POSS nanohybrid appears no obvious destruction under the present adsorption-desorption conditions (Fig. S8B, C and D).

\subsection{Adsorption and removal dyes from real textile wastewater}

The developed dye removal approach by cross-linked POSS nanohybrid is further applied for the treatment of real textile wastewater (kindly donated by Prof. Xueyan Wang at Xi'an Polytechnic University). The textile wastewater is alkaline in nature ( $\mathrm{pH}$ 11.2 \pm 0.2 ) with a high chemical oxygen demand (COD) of $1704 \mathrm{mg} \mathrm{L}^{-1}$ (measured by using DR3000 water analyzer from Shanghai Xinrui Instrument \& Meters. Co. Ltd., China). The adsorption and removal process is carried out under optimized condition (adsorbent dosage $=5 \mathrm{mg}$, adsorption time $=60 \mathrm{~min}, \mathrm{pH}$ value $=6$, wastewater volume $=3$ $\mathrm{mL}$ ) and the supernatant is collected to test the removal performance. The residual COD value of wastewater after adsorption process significantly decreases to be $378 \mathrm{mg}$

$\mathrm{L}^{-1}$, giving rise to a COD removal efficiency of $77.8 \%$. Meanwhile, a decolourization ratio of $c a .93 \%$ is also obtained by monitoring the absorbance variations of initial and residual wastewater. The real sample test demonstrates that cross-linked POSS nanohybrid shows favorable practical applicability in the field of dyeing wastewater treatment.

\section{Conclusions}

In this work, we prepare a novel cross-linked POSS nanohybrid as adsorbent for 
selective adsorption and removal acidic dyes from aqueous solutions. The synthesis of cross-linked POSS nanohybrid is taken place in aqueous solution at room temperature by using OA-POSS as building blocks and glutaraldehyde as dual functional cross-linking bridge. The introduction of glutaraldehyde densely connects each OA-POSS molecule together and effectively increases the insolubility of the nanohybrid, thus overcoming the high solubility of OA-POSS in water and facilitating the operation of phase separation. Cross-linked POSS nanohybrid exhibits selective adsorption ability toward acidic dyes, i.e., MO in this case, through strong electrostatic interactions. The adsorption/removal of MO from aqueous solution could be completed in a short time with a favorable adsorption capacity and high removal efficiency by comparing with other adsorbents in previous works. Furthermore, the good removal performance (COD removal efficiency of $77.8 \%$ and decolourization ratio of $93 \%$ ) after treatment of textile wastewater demonstrates that cross-linked POSS nanohybrid appears to be a promising material in the field of dye removal. Overall, the developed cross-linking strategy successfully opens a new avenue for the application of POSS-based materials as adsorbents in dyeing wastewater treatment. Moreover, the residual reactive groups (amino groups) after cross-linking reaction might be further functionalized to improve the selectivity towards other targeted molecules, which would be employed in various adsorption and separation fields in the future. 


\section{Acknowledgement}

The authors gratefully acknowledge financial support from the National Science Foundation of China (Grant Numbers: 21605122, 21545007, and 21505104), and the Foundation of Key Laboratory in Shaanxi Province (Grant Numbers: 16JS116, and 15JS115). 


\section{Reference}

1. L.J. Zhang, P. Hu, J. Wang, Q. Liu, R.H. Huang, Adsorption of methyl orange (MO) by Zr (IV)-immobilized cross-linked chitosan/bentonite composite, Int. J. Biol. Macromol. 81 (2015) 818-827.

2. Y.P. Chang, C.L. Ren, Q. Yang, Z.Y. Zhang, L.J. Dong, X.G. Chen, D.S. Xue, Preparation and characterization of hexadecyl functionalized magnetic silica nanoparticles and its application in Rhodamine 6G removal, Appl. Surf. Sci. 257 (2011) 8610-8616.

3. A.U. Metin, H. Ciftci, E. Alver, Efficient removal of acidic dye using low-cost biocomposite beads, Ind. Eng. Chem. Res. 52 (2013) 10569-10581.

4. V.K. Balakrishnan, S. Shirin, A.M. Aman, S.R. de Solla, J. Mathieu-Denoncourt, V.S. Langlois, Genotoxic and carcinogenic products arising from reductive transformations of the azo dye, Disperse Yellow 7, Chemosphere 146 (2016) 206-215.

5. S. Marie, H.S. Heinz, F. Eva, H. Petr, M. Vaclav, Enzymes oxidizing the azo dye 1-phenylazo-2-naphthol (Sudan I) and their contribution to its genotoxicty and carcinogenicity, Curr. Drug Metab. 15 (2014) 829-840.

6. L. Cheng, M.Y. Wei, L.H. Huang, F. Pan, D.S. Xia, X.X. Li, A.H. Xu, Efficient $\mathrm{H}_{2} \mathrm{O}_{2}$ oxidation of organic dyes catalyzed by simple copper (II) ions in bicarbonate aqueous solution, Ind. Eng. Chem. Res. 53 (2014) 3478-3485. 
7. D.L. Zhao, X. Yang, C.L. Chen, X.K. Wang, Enhanced photocatalytic degradation of methylene blue on multiwalled carbon nanotubes- $\mathrm{TiO}_{2}$, J. Colloid Interface Sci. 398 (2013) 234-239.

8. A.K. Verma, R.R. Dash, P. Bhunia, A review on chemical coagulation/flocculation technologies for removal of colour from textile wastewaters, J. Environ. Manage. 93 (2012) 154-168.

9. K. Sarayu, S. Sandhya, Current technologies for biological treatment of textile wastewater-a review, Appl. Biochem. Biotech. 167 (2012) 645-661.

10. Y.P. Zheng, G.H. Yao, Q.B. Cheng, S.C. Yu, M.H. Liu, C.J. Gao, Positively charged thin-film composite hollow fiber nanofiltration membrane for the removal of cationic dyes through submerged filtration, Desalination 328 (2013) 42-50.

11. M.T. Yagub, T.K. Sen, S. Afroze, H.M. Ang, Dye and its removal from aqueous solution by adsorption: a review, Adv. Colloid Interface Sci. 209 (2014) 172-184.

12. Y. Liu, C. Luo, J. Sun, H.Z. Li, Z.B. Sun, S.Q. Yan, Enhanced adsorption removal of methyl orange from aqueous solution by nanostructured proton-containing $\delta-\mathrm{MnO}_{2}$, J. Mater. Chem. A, 3 (2015) 5674-5682.

13. F. Liu, Y. Zhang, L. Xu, W.A. Zhang, Morphology-controlled self-assembly of an organic/inorganic hybrid porphyrin derivative containing polyhedral oligomeric silsesquioxane (POSS), Chem. Eur. J. 21 (2015) 5540-5547.

14. G.Z. Li, L.C. Wang, H.L. Ni, C.U. Pittman Jr., Polyhedral oligomeric silsesquioxane (POSS) polymers and copolymers: a review, J. Inorg. Organomet. 
Polym. 11 (2001) 123-154.

15. J. Wu, P.T. Mather, POSS polymers: physical properties and biomaterials applications, J. Macromol. Sci. Polym. Rev. 49 (2009) 25-63.

16. T.F. Zhang, J.Z. Wang, M.X. Zhou, L. Ma, G.Z. Yin, G.X. Chen, Q.F. Li, Influence of polyhedral oligomeric silsesquioxane (POSS) on blue light-emitting materials for OLED, Tetrahedron 70 (2014) 2478-2486.

17. W.C. Zhang, X.M. Li, R.J. Yang, Flame retardant mechanisms of phosphorus containing polyhedral oligomeric silsesquioxane (DOPO-POSS) in polycarbonate composites, J. Appl. Polym. Sci. 124 (2012) 1848-1857.

18. H. Yahyaei, M. Mohseni, H. Ghanbari, M. Messori, Synthesis and characterization of polyhedral oligomeric titanized silsesquioxane: a new biocompatible cage like molecule for biomedical application, Mater. Sci. Eng. C 61 (2016) 293-300.

19. J.J. Ou, Z.S. Liu, H.W. Wang, H. Lin, J. Dong, H.F. Zou, Recent development of hybrid organic-silica monolithic columns in CEC and capillary LC, Electrophoresis 36 (2015) 62-75.

20. K. Tanaka, Y. Chujo, Advanced functional materials based on polyhedral oligomeric silsesquioxane (POSS), J. Mater. Chem. 22 (2012) 1733-1746.

21. Q.W. Li, W.B. Zhang, I.F. Hsieh, G.L. Zhang, Y. Cao, X.P. Li, C. Wesdemiotis, B. Lotz, H.M. Xiong, S.Z.D. Cheng, Breaking symmetry toward nonspherical Janus particles based on polyhedral oligomeric silsesquioxane: molecular design, "click" synthesis, and hierarchical structure, J. Am. Chem. Soc. 133 (2011) 10712-10715. 
22. B. Trastoy, D.A. Bonsor, M.E. Perez-Ojeda, M.L. Jimeno, A. Mendez-Ardoy, J.M.G. Fernandez, E.J. Sundberg, J.L. Chiara, Synthesis and biophysical study of disassembling nanohybrid bioconjugates with a cubic octasilsesquioxane core, Adv. Funct. Mater. 22 (2012) 3191-3201.

23. Z.X. Zhou, Z.R. Lu, Dendritic nanoglobules with polyhedral oligomeric silsesquioxane core and their biomedical applications, Nanomedicine 9 (2014) 2387-2401.

24. S. Fabritz, S. Horner, O. Avrutina, H. Kolmar, Bioconjugation on cube-octameric silsesquioxanes, Org. Biomol. Chem. 11 (2013) 2224-2236.

25. X. Zhao, W.J. Zhang, Y.Z. Wu, H.Z. Liu, X.P. Hao, Facile fabrication of OA-POSS modified near-infrared-emitting CdSeTe alloyed quantum dots and their bioapplications, New J. Chem. 38 (2014) 3242-3249.

26. W.J. Wang, X. Hai, Q.X. Mao, M.L. Chen, J.H. Wang, polyhedral oligomeric silsesquioxane functionalized carbon dots for cell imaging, ACS Appl. Mater. Interfaces 7 (2015) 16609-16616.

27. K. Tanaka, W. Ohashi, N. Kitamura, Y. Chujo, Reductive glutathione-responsive molecular release using water-soluble POSS network polymers, Bull. Chem. Soc. Jpn. 84 (2011) 612-616.

28. J.W. Jeon, T. Kakuta, K. Tanaka, Y. Chujo, Facile design of organic-inorganic hybrid gels for molecular recognition of nucleoside triphosphates, Bioorg. Med. Chem. Lett. 25 (2015) 2050-2055. 
29. Y. Kim, K. Koh, M.F. Roll, R.M. Laine, A.J. Matzger, Porous networks assembled from octaphenylsilsesquioxane building blocks, Macromolecules 43 (2010) 6995-7000.

30. I. NIshchang, O. Bruggemann, I. Teasdale, Facile, single-step preparation of versatile, high-surface-area, hierarchically structured hybrid materials, Angew. Chem. Int. Ed. 50 (2011) 4592-4596.

31. Y.C. Qin, H.B. Ren, F.H. Zhu, L. Zhang, C.W. Shang, Z.J. Wei, M.M. Luo, Preparation of POSS-based organic-inorganic hybrid mesoporous materials networks through Schiff base chemistry, Eur. Polym. J. 47 (2011) 853-860.

32. Z.P. Zhang, G.Z. Liang, T.L. Lu, Synthesis and characterization of cage octa(aminopropylsilsesquioxane), J. Appl. Polym. Sci. 103 (2007) 2608-2614.

33. F. Rouquerol, J. Rouquerol, K.S.W. Sing, Adsorption by powders and porous solids: principles, methodology and applications, Academic Press, London, 1999, pp. 18-20.

34. H.L. Cai, K. Xu, X. Liu, Z. Fu, M.C. Chen, A facile synthesis of octa(carboxyphenyl)silsesquioxane, Dalton Trans. 41 (2012) 6919-6921.

35. J.W. Liu, T. Yang, S. Chen, X.W. Chen, J.H. Wang, Nickel chelating functionalization of graphene composite for metal affinity membrane isolation of lysozyme, J. Mater. Chem. B 1 (2013) 810-818.

36. M. Campinas, M.J. Rosa, The ionic strength effect on microcystin and natural organic matter surrogate adsorption onto PAC, J. Colloid Interface Sci. 299 (2006) 
$520-529$.

37. H.X. Guo, F. Lin, J.H. Chen, F.M. Li, W. Weng, Metal-organic framework MIL-125(Ti) for efficient adsorptive removal of Rhodamine B from aqueous solution, Appl. Organometal. Chem. 29 (2015) 12-19.

38. A. Roy, B. Adhikari, S.B. Majumder, Equilibrium, kinetic, and thermodynamics studies of azo dye adsorption from aqueous solution by chemically modified lignocellulosic jute fiber, Ind. Eng. Chem. Res. 52 (2013) 6502-6512.

39. R.I. Yousef, B. EI-Eswed, A.H. AI-Muhtaseb, Adsorption characteristics of natural zeolites as solid adsorbent for phenol removal from aqueous solutions: kinetics, mechanism, and thermodynamics studies, Chem. Eng. J. 171 (2011) 1143-1149.

40. J. Hu, D.D. Shao, C.L. Chen, G.D. Sheng, X.M. Ren, X.K. Wang. Removal of 1-naphthylamine from aqueous solution by multiwall carbon nanotubes/iron oxides/cyclodextrin composite, J. Hazard. Mater. 185 (2011) 463-471.

41. Z. Aksu, G. Donmez, Binary biosorption of cadmium (II) and nickel (II) onto dried Chlorella vulgaris: co-ion effect on mono-component isotherm parameters, Process Biochem. 41 (2006) 860-868.

42. R. Donat, A. Akdogan, E. Erdem, H. Cetisli, Thermodynamics of $\mathrm{Pb}^{2+}$ and $\mathrm{Ni}^{2+}$ adsorption onto natural bentonite from aqueous solutions, J. Colloid Interface Sci. $286(2005) 43-52$.

43. R.A.K. Rao, M.A. Khan, F. Rehman, Utilization of fennel biomass (Foeniculum vulgari) a medicinal herb for the biosorption of Cd (II) from aqueous phase, Chem. 
Eng. J. 156 (2010) 106-113.

44. D. Robati, B.M.M. Rajabi, O. Moradi, I. Tyagi, S. Agarwal, V.K. Gupta, Removal of hazardous dyes-BR12 and methyl orange using graphene oxide as an adsorbent from aqueous phase, Chem. Eng. J. 284 (2016) 687-697.

45. Y.J. Yao, B. He, F.F. Xu, X.F. Chen, Equilibrium and kinetic studies of methyl orange adsorption on multiwalled carbon nanotubes, Chem. Eng. J. 170 (2011) 82-89.

46. Y. Haldorai, J.J. Shim, An efficient removal of methyl orange dye from aqueous solution by adsorption onto chitosan/MgO composite: a novel reusable adsorbent, Appl. Surf. Sci. 292 (2014) 447-453.

47. H.Y. Zhu, R. Jiang, L. Xiao, G.M. Zeng, Preparation, characterization, adsorption kinetics and thermodynamics of novel magnetic chitosan enwrapping nanosized $\gamma-\mathrm{Fe}_{2} \mathrm{O}_{3}$ and multi-walled carbon nanotubes with enhanced adsorption properties for methyl orange, Bioresour. Technol. 101 (2010) 5063-5069.

48. W. Cheah, S. Hosseini, M.A. Khan, T.G. Chuah, T.S.Y. Choog, Acid modified carbon coated monolith for methyl orange adsorption, Chem. Eng. J. 215-216 (2013) 747-754.

49. S.H. Chen, J. Zhang, C.L. Zhang, Q.Y. Yue, Y. Li, C. Li, Equilibrium and kinetic studies of methyl orange and methyl violet adsorption on activated carbon derived from Phragmites australis, Desalination 252 (2010) 149-156.

50. M. Arshadi, M. Mehravar, M.J. Amiri, A.R. Faraji, Synthesis and adsorption 
characteristics of an heterogenized manganese nanoadsorbent towards methyl orange, J. Colloid Interface Sci. 440 (2015) 189-197.

51. Y. Zhao, H.L. Chen, J. Li, C.L. Chen, Hierarchical MWCNTs/Fe ${ }_{3} \mathrm{O}_{4} / \mathrm{PANI}$ magnetic composite as adsorbent for methyl orange removal, J. Colloid Interface Sci. 450 (2015) 189-195.

52. J. Li, Z.Y. Shao, C.L. Chen, X.K. Wang. Hierarchical GOs/ $/ \mathrm{Fe}_{3} \mathrm{O}_{4} / \mathrm{PANI}$ magnetic composites as adsorbent for ionic dye pollution treatment, RSC Adv. 4 (2014) 38192-38198.

53. J.Q. Jiang, C.X. Yang, X.P. Yan, Zeolitic imidazolate framework-8 for fast adsorption and removal of benzotriazoles from aqueous solution, ACS Appl. Mater. Interface 5 (2013) 9837-9842.

54. X. Yang, C.L. Chen, J.X. Li, G.X. Zhao, X.M. Ren, X.K. Wang, Graphene oxide-iron oxide and reduced graphene oxide-iron oxide hybrid materials for the removal of organic and inorganic pollutants, RSC Adv. 2 (2012) 8821-8826. 


\section{Figure Legends}

Scheme 1. The synthesis procedure of cross-linked POSS nanohybrid and adsorption mechanism of MO on the nanohybrid.

Fig. 1. FT-IR spectra (A) and solid-state ${ }^{29} \mathrm{Si}$ NMR spectra (B) of (a) OA-POSS, and (b) cross-linked POSS nanohybrid.

Fig. 2. SEM (A) and TEM (B) images of cross-linked POSS nanohybrid.

Fig. 3. Effect of contact time on MO adsorption onto the cross-linked POSS nanohybrid at three different concentrations i.e., 50, 100, $200 \mathrm{mg} \mathrm{L}^{-1}$, respectively. Adsorption experiments are performed in $3 \mathrm{~mL}$ of $\mathrm{MO}$ neutral solution with $5 \mathrm{mg}$ sorbent at $293 \mathrm{~K}$.

Fig. 4. Adsorption isotherm curves of MO onto cross-linked POSS nanohybrid at five different temperatures. Adsorption condition: sorbent dosage: $5 \mathrm{mg}$; contact time: 60 min; $\mathrm{pH}$ : 6; MO concentration range and volume are $10-700 \mathrm{mg} \mathrm{L}^{-1}$ and $3.0 \mathrm{~mL}$, respectively.

Fig. 5. (A) various kinds of eluents for MO desorption from the cross-linked POSS nanohybrid. 1: acetonitrile, 2: methanol, 3: methanol/ $\mathrm{NaOH}\left(9 / 1, \mathrm{v} / \mathrm{v}, 0.1 \mathrm{~mol} \mathrm{~L}^{-1}\right.$ 
$\mathrm{NaOH}$ ); (B) Adsorption results for investigating regeneration of the cross-linked POSS nanohybrid in successive processing of adsorption-desorption of MO species. Adsorption condition: sorbent dosage: $5 \mathrm{mg}$; adsorption time: $60 \mathrm{~min}$; $\mathrm{pH}$ : 6; $\mathrm{MO}$ concentration and volume are $100 \mathrm{mg} \mathrm{L}^{-1}$ and $3.0 \mathrm{~mL}$, respectively. Desorption condition: elution time: $90 \mathrm{~min}$, eluent volume: $1.0 \mathrm{~mL}$. 


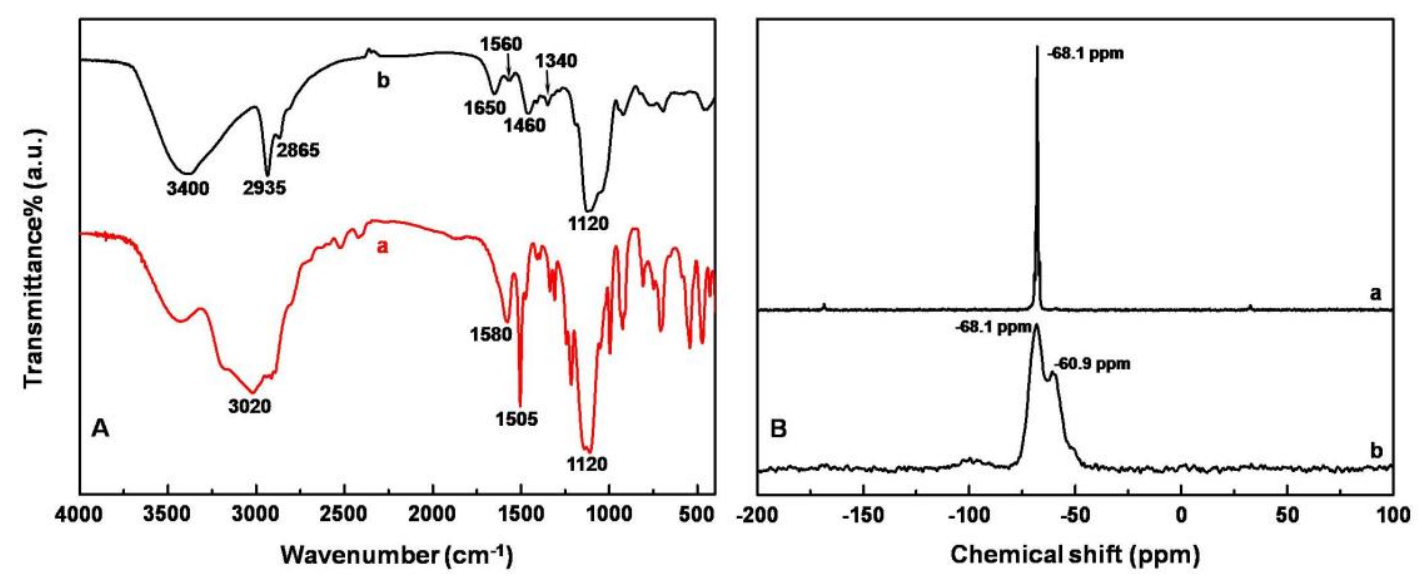




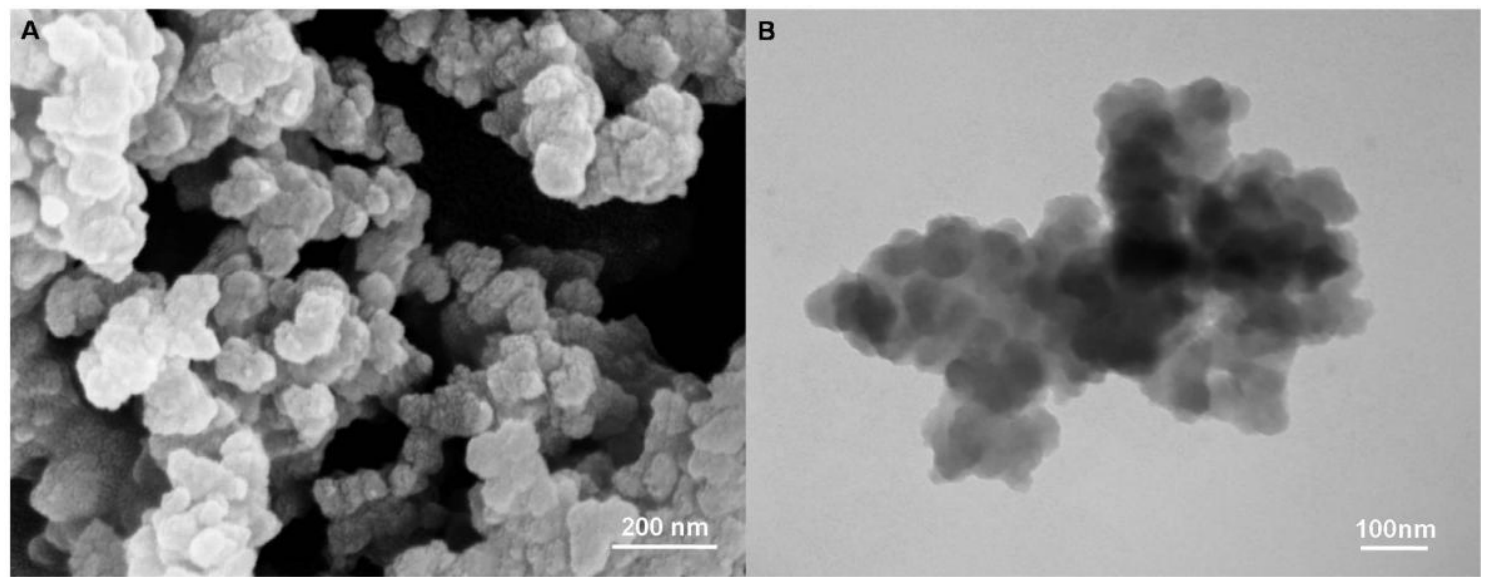




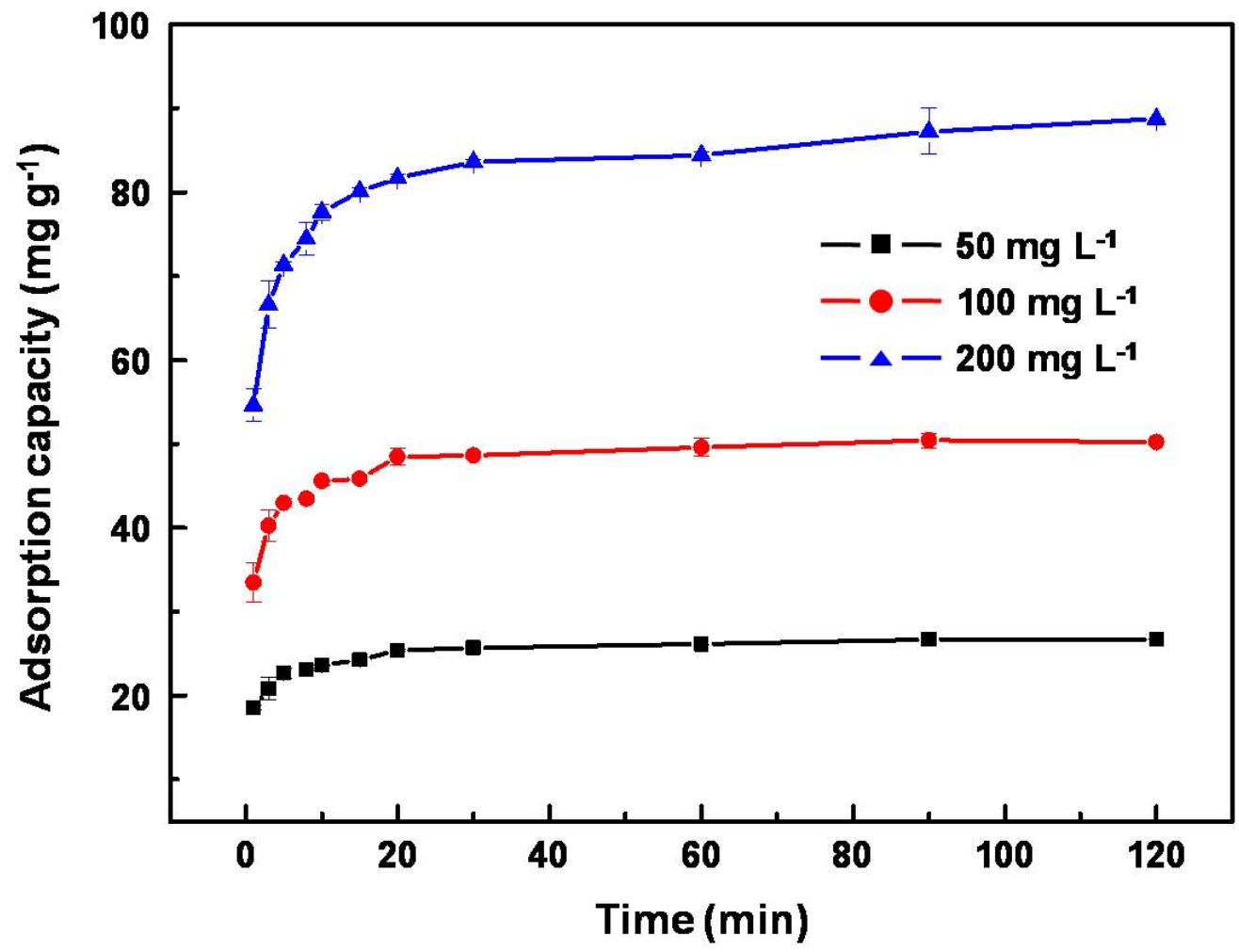




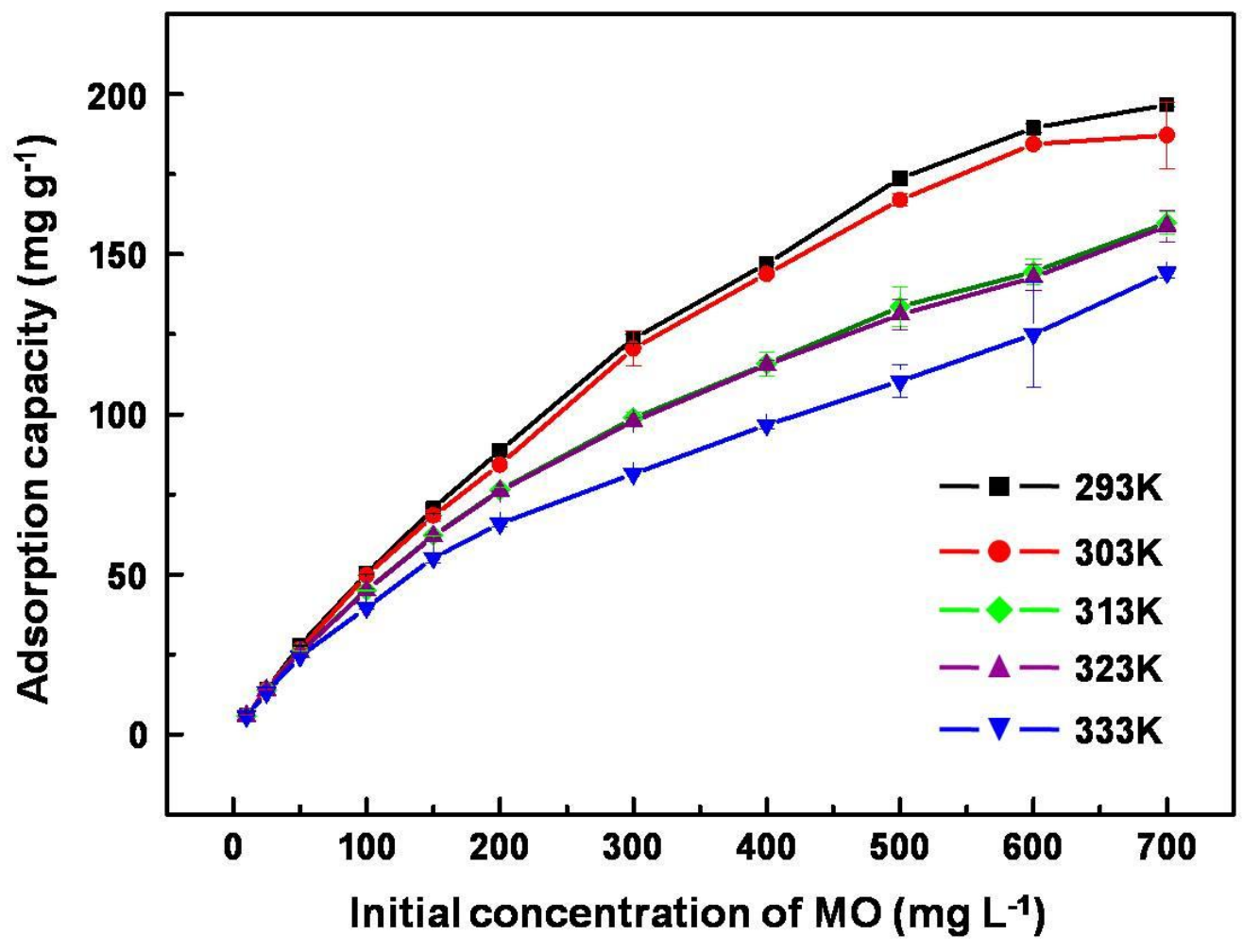



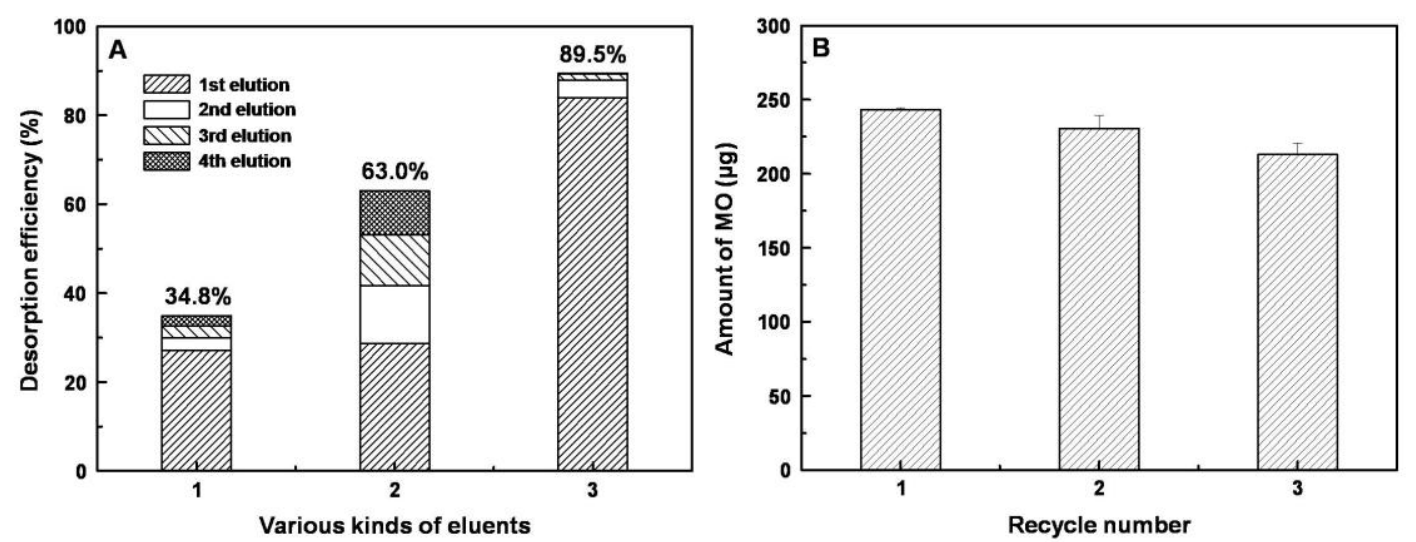
Table 1 Calculated parameters of pseudo-first-order and pseudo-second-order kinetic model for MO adsorption under different initial concentrations.

\begin{tabular}{|c|c|c|c|c|c|c|c|}
\hline \multirow{2}{*}{$\begin{array}{c}c_{0} \\
(\mathrm{mg} \\
\left.\mathrm{L}^{-1}\right)\end{array}$} & \multirow{2}{*}{$\begin{array}{c}q_{e, e x} \\
p \\
(\mathrm{mg} \\
\left.\mathrm{g}^{-1}\right)^{\mathrm{a}}\end{array}$} & \multicolumn{3}{|c|}{ Pseudo-first-order kinetic model } & \multicolumn{3}{|c|}{ Pseudo-second-order kinetic model } \\
\hline & & $\begin{array}{l}q_{e, c a l}(\mathrm{mg} \\
\left.\mathrm{g}^{-1}\right)^{\mathrm{b}}\end{array}$ & $\begin{array}{r}k_{1} \\
\left(\min ^{-1}\right)\end{array}$ & $R^{2}$ & $\begin{array}{r}q_{e, c a l} \\
\left(\mathrm{mg} \mathrm{g}^{-1}\right)\end{array}$ & $\begin{array}{l}k_{2}\left(\mathrm{~g} \mathrm{mg}^{-1}\right. \\
\left.\min ^{-1}\right)\end{array}$ & ${ }^{2} R$ \\
\hline 50 & 27.7 & 6.0 & $\begin{array}{l}0.028 \\
0\end{array}$ & $\begin{array}{r}0 . \\
7518\end{array}$ & 26.9 & 0.0313 & $\begin{array}{r}0 . \\
9998\end{array}$ \\
\hline 100 & 50.4 & 13.7 & $\begin{array}{l}0.019 \\
2\end{array}$ & $\begin{array}{r}0 . \\
6303\end{array}$ & 50.7 & 0.0188 & $\begin{array}{r}0 . \\
9998\end{array}$ \\
\hline 200 & 88.7 & 32.6 & $\begin{array}{l}0.016 \\
4\end{array}$ & $\begin{array}{r}0 . \\
6760\end{array}$ & 88.9 & 0.0075 & $\begin{array}{r}0 . \\
9996\end{array}$ \\
\hline
\end{tabular}

${ }^{\mathrm{a}}$ : Equilibrium adsorption capacity obtained from experiment.

${ }^{\mathrm{b}}$ : Equilibrium adsorption capacity calculated according to kinetic models 
Table 2 Adsorption parameters of three isotherm models for MO onto the cross-linked POSS nanohybrid at different temperature.

\begin{tabular}{|c|c|c|c|c|}
\hline \multirow{2}{*}{$\begin{array}{l}\text { Isotherm } \\
\text { model }\end{array}$} & \multirow{2}{*}{$\begin{array}{l}\text { Temperatur } \\
\text { e } \\
\text { (K) }\end{array}$} & \multicolumn{3}{|c|}{ Model parameters } \\
\hline & & $q_{m}\left(\mathrm{mg} \mathrm{g}^{-1}\right)$ & $\begin{array}{r}b(\mathrm{~L} \\
\left.\mathrm{mg}^{-1}\right)\end{array}$ & $R^{2}$ \\
\hline \multirow{5}{*}{ Langmuir } & 293 & 237.5 & 0.0127 & 0.9967 \\
\hline & 303 & 229.9 & 0.0118 & 0.9952 \\
\hline & 313 & 188.7 & 0.0096 & 0.9922 \\
\hline & 323 & 186.6 & 0.0095 & 0.9917 \\
\hline & 333 & 142.9 & 0.0111 & 0.9818 \\
\hline \multirow{6}{*}{ Freundlich } & & $\begin{array}{c}K_{F}\left(\mathrm{mg}^{1-1 / \mathrm{n}} \mathrm{L}^{1 / \mathrm{n}}\right. \\
\left.\mathrm{g}^{-1}\right)\end{array}$ & $1 / n$ & $R^{2}$ \\
\hline & 293 & 13.20 & 0.48 & 0.9960 \\
\hline & 303 & 11.50 & 0.49 & 0.9972 \\
\hline & 313 & 10.42 & 0.45 & 0.9991 \\
\hline & 323 & 10.35 & 0.45 & 0.9991 \\
\hline & 333 & 8.11 & 0.46 & 0.9982 \\
\hline \multirow{6}{*}{ D-R } & & $q_{D-R}\left(\mathrm{mg} \mathrm{g}^{-1}\right)$ & $\begin{array}{l}E(\mathrm{~kJ} \\
\left.\mathrm{mol}^{-1}\right)\end{array}$ & $R^{2}$ \\
\hline & 293 & 135.7 & 6.93 & 0.9514 \\
\hline & 303 & 131.3 & 6.86 & 0.9522 \\
\hline & 313 & 111.5 & 6.71 & 0.9663 \\
\hline & 323 & 109.8 & 6.98 & 0.9646 \\
\hline & 333 & 93.7 & 6.87 & 0.9589 \\
\hline
\end{tabular}


Table 3 Comparison of the MO removal performance of cross-linked POSS nanohybrid with other adsorbents.

\begin{tabular}{|c|c|c|c|c|c|c|c|c|}
\hline Adsorbent & $\begin{array}{c}\text { Adsorpti } \\
\text { on capacity } \\
\left(\mathrm{mg} \mathrm{g}^{-1}\right)\end{array}$ & $\mathrm{pH}$ & $\begin{array}{l}\text { Tempera } \\
\text { ture } \\
\quad(\mathrm{K})\end{array}$ & $\begin{array}{l}\text { Contact } \\
\text { time } \\
(\min )\end{array}$ & $\begin{array}{c}\text { MO } \\
\text { initial } \\
\text { concentration } \\
\left(\mathrm{mg} \mathrm{L}^{-1}\right)\end{array}$ & $\begin{array}{r}\text { Adso } \\
\text { rbent } \\
\text { dosage } \\
(\mathrm{g} \\
\left.\mathrm{L}^{-1}\right)\end{array}$ & $\begin{array}{l}\text { Remo } \\
\text { val } \\
\text { efficiency } \\
(\%)\end{array}$ & Ref. \\
\hline Graphene oxide & 16.8 & 3.0 & 298 & 100 & 0.2 & 1.0 & 48 & 44 \\
\hline Multi-walled carbon nanotubes & 51.7 & $\begin{array}{l}\text { neut } \\
\text { ral }\end{array}$ & 298 & 120 & 20 & 0.3 & 43.5 & 45 \\
\hline Chitosan/MgO composite & 60.0 & 8.0 & $\mathrm{RT}^{\mathrm{a}}$ & 5 & 100 & 5.0 & 98 & 46 \\
\hline Chitosan $/ \gamma-\mathrm{Fe}_{2} \mathrm{O}_{3} / \mathrm{MCNTs}$ & 66.1 & $\begin{array}{l}\text { neut } \\
\text { ral }\end{array}$ & 297 & 180 & 20 & 0.6 & 94.6 & 47 \\
\hline $\begin{array}{l}\text { Acid modified carbon coated } \\
\text { monolith }\end{array}$ & 147.1 & 6.0 & 303 & 4320 & 250 & 10 & 35.2 & 48 \\
\hline $\begin{array}{l}\text { Phragmites australis activation } \\
\text { carbon }\end{array}$ & 238.1 & $\begin{array}{l}\text { neut } \\
\text { ral }\end{array}$ & 283 & 240 & 50 & 0.5 & 98 & 49 \\
\hline Si/Al-PAEA=SA@MnNP & 246.8 & 8.0 & 298 & 20 & 150 & 5.0 & 89.9 & 50 \\
\hline $\mathrm{MCNTs} / \mathrm{Fe}_{3} \mathrm{O}_{4} / \mathrm{PANI}$ & 446.25 & 4.0 & 298 & 750 & 150 & 0.4 & $67.2^{b}$ & 51 \\
\hline $\mathrm{GOs} / \mathrm{Fe}_{3} \mathrm{O}_{4} / \mathrm{PANI}$ & 585.02 & 4.0 & 298 & 300 & 150 & 0.4 & $82.7^{\mathrm{b}}$ & 52 \\
\hline Cross-linked POSS nanohybrid & 237.5 & 6.0 & 293 & 60 & 100 & 1.67 & 85 & $\begin{array}{l}\text { This } \\
\text { work }\end{array}$ \\
\hline
\end{tabular}

${ }^{\mathrm{a}}$ : room temperature

b. estimated value 


\section{Graphical Abstract}

Cross-linked polyhedral oligomeric silsesquioxane (POSS) nanohybrid is prepared by using octa-amino POSS as building blocks and glutaraldehyde as dual functional bridge, and further employed as a novel adsorbent for selective adsorption and removal of acidic dyes from aqueous solution.

Synthesis

Selective adsorption

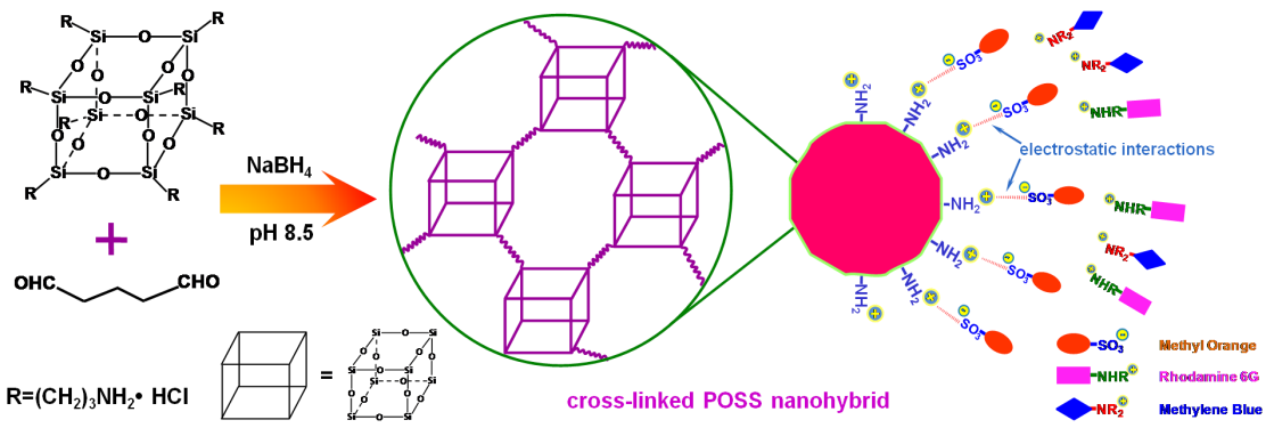

